\title{
Photo-response Assisted Enantiomer Separations on an Azobenzene-modified $\gamma$-Cyclodextrin Stationary Phase in Micro-HPLC
}

\author{
Tatsuro NaKagama, ${ }^{\dagger}$ Atsutoshi Yamaguchi, Kazuya Hirasawa, Koji Yoshida, \\ Katsumi UCHIYAMA, and Toshiyuki Ново \\ Division of Applied Chemistry, Graduate School of Engineering, Tokyo Metropolitan University, \\ 1-1 Minamiosawa, Hachioji, Tokyo 192-0397, Japan
}

\begin{abstract}
The photo-responses of the retention and enantioseparation of several optical isomers were evaluated using an azobenzene-modified $\gamma$-cyclodextrin stationary phase (Az $\gamma$-CDSP) in micro-HPLC. UV light irradiation induced a decrease in the retention and the chiral selectivity for $N$-(3,5-dinitrobenzoyl)-1-phenylethylamine (DNBPEA) and $N$-(3,5dinitrobenzoyl)-1-(1-naphtylethyl)amine (DNBNEA), while an increase was induced for dansylphenylalanine (DnsPhe) using a mixture of methanol and aqueous phosphate buffer as the mobile phase. No changes in the retention and the enantiomer separation of benzoin were observed with UV light irradiation. The retention behaviors were recovered by visible-light irradiation. It was speculated that the main factor of the change in the retention behavior was a change in the $\pi-\pi$ interaction due to the azobenzene moiety of the stationary phase with photo-irradiation. Comparing the retention behavior before and after UV light irradiation, a suitable condition for obtaining a better resolution and enantiomer separation would be chosen using Az $\gamma$-CDSP.
\end{abstract}

(Received October 15, 2001; Accepted November 30, 2001)

Micro-HPLC has become an important separation technique because it has many advantages, such as low consumption of organic and toxic solvents, applicability of expensive mobile phases, and easier coupling to a mass spectrometer. To make the best use these advantages, however, some special instrumentations and techniques are required.

Those techniques, such as solvent- and/or temperatureprogramming elution or column switching, which are familiar with ordinary HPLC, can also be applied to micro-HPLC with some modification. To the best of our knowledge, solventgradient elution and column switching require special instruments, and the temperature-gradient elution technique is slightly effective to control of the retention order.

To change the selectivity of the LC column, the idea of on-site switching of the stationary phase by light irradiation has been proposed. $^{1-5}$ It is based on a reversibly photo-responsive stationary phase, whose selectivity can be switched from outside the column. Various advantages can be imagined: some pure solvents can be used as the mobile phase, the property of the stationary phase can be adjusted to the analytes due to the continuous change of the retention behaviors, and it can be applied to micro-HPLC, CEC and other separation systems. In order to realize these ideas, several stationary phases using azobenzene derivatives have been designed and prepared. ${ }^{4,5}$ In our previous study, an azobenzene-modified $\gamma$-cyclodextrin stationary phase (Az $\gamma$-CDSP) was prepared and the photoresponses of the retentions of the perylene and pentacene were investigated in micro-HPLC. ${ }^{5}$ Changes in the retention specific to solutes were observed with Az $\gamma$-CDSP by light irradiation.

† To whom correspondence should be addressed.

E-mail: nakagama-tatsuro@c.metro-u.ac.jp
Native and modified $\beta$ - and $\gamma$-CD stationary phases are also reported to be useful for the resolution of dansylated (Dns) $\alpha$ amino acids ${ }^{6-10}$ and other enantiomers. ${ }^{11,12}$ The separations of the enantiomers of Dns- $\alpha$-amino acids utilizing natural and modified- $\beta$-CD or $\gamma$-CD bonded phases have been investigated, and a natural $\gamma-\mathrm{CD}$ bonded phase was proved to be very useful for the separation of not only the enantiomers, but also the homologues of Dns-amino acids. ${ }^{10}$ The separation can be easily optimized by changing the mobile-phase parameters, such as the $\mathrm{pH}$ and ionic strength, as well as the type and concentration of organic modifier in conventional HPLC. ${ }^{7-10}$ However, it would be rather difficult to change these separation conditions in micro-separation systems.

In this work, a micro-HPLC system for chiral separation using Az $\gamma$-CDSP was investigated. The separation can be optimized by light irradiation without changing the mobile phase or the column, itself. The photo-responses of the retentions and the separations of several optical isomers were evaluated.

\section{Experimental}

Preparation of stationary phases (Fig. 1)

Az $\gamma$-CDSP was prepared by a previously reported method. 5 An azobenzene-modified $\gamma-\mathrm{CD}(\mathrm{Az} \gamma-\mathrm{CD})$ was first synthesized by the esterification of $\gamma$-CD with $p$-phenylazobenzoyl chloride. It was confirmed that one group of the azobenzene moiety was modified on a $\gamma$-CD molecule by measuring the carbon content by elemental analysis (found, $47.85 \%$; calculated, $48.09 \%$ for the monohydrate $\left.{ }^{13}\right)$.

Az $\gamma-C D$ was then chemically bonded on porous silica (Develosil silica, 100-5; mean particle size, $5 \mu \mathrm{m}$; mean pore 

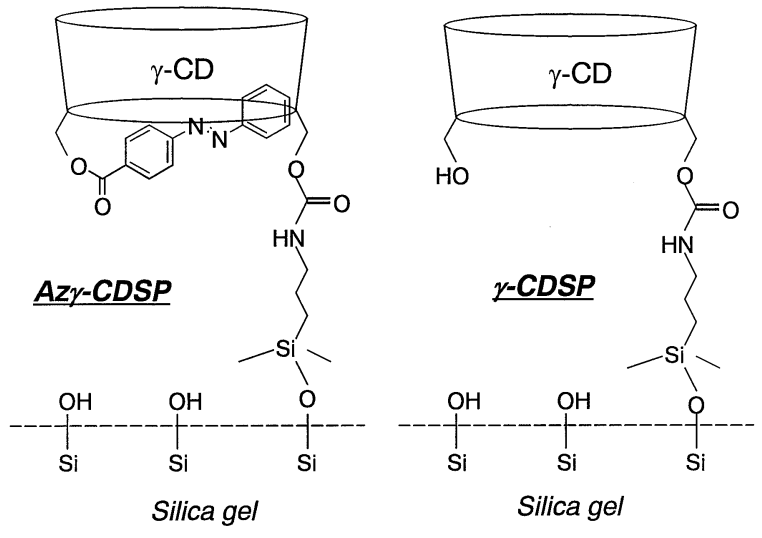

Fig. 1 Azobenzene-modified and native $\gamma$-CD stationary phases (Az $\gamma$-CDSP and $\gamma$-CDSP).

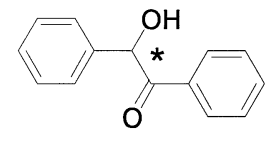

Benzoin

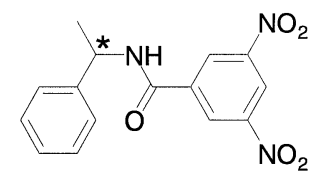

DNBPEA

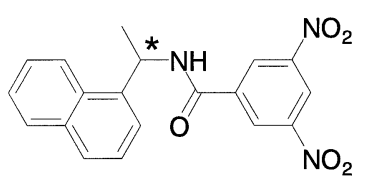

DNBNEA

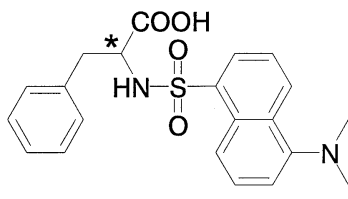

DnsPhe
Fig. 2 Test enantiomers in this study.

size, $100 \AA$; and specific surface area, $350 \mathrm{~m}^{2} / \mathrm{g}$ ) purchased from the Nomura Chemical Co. (Aichi, Japan), using 3isocyanatopropyltriethoxysilane. The amount of Az $\gamma-\mathrm{CD}$ moieties on the silica support was estimated from the carbon contents $(10.7 \%)$ to be 0.24 groups $/ \mathrm{nm}^{2}$.

A native $\gamma$-CD stationary phase $(\gamma$-CDSP) was prepared by a method similar to that described previously ${ }^{5}$ for Az $\gamma$-CDSP (0.18 groups $\left./ \mathrm{nm}^{2}\right)$.

\section{Samples (Fig. 2)}

Four enantiomers $(R-$ and $S$ - $N$-(3,5-dinitrobenzoyl)-1phenylethylamine (DNBPEA), $R$ - and $S$ - $N$-(3,5-dinitrobenzoyl)1-(1-naphtyl)ethylamine (DNBNEA), dansyl-L-and-D,Lphenylalanine (DnsPhe), and $R$ - and $S$-benzoin) were chosen and tested in this study.

\section{Instrumentation}

The micro-LC system used in this study consisted of a Shimadzu (Kyoto, Japan) Model LC-10AD high-pressure pump, an LC Packings (San Francisco, CA, USA) IC-400-VAR stream splitter, a Reodyne (Cotati, CA, USA) Model 7520 sample injector $(0.2 \mu \mathrm{l})$, a Shimadzu CTO-10AC column oven, a light source, a JASCO (Tokyo, Japan) CE-971UV detector and a Shimadzu C-R7Aplus integrator. A mixture of methanol and aqueous phosphate buffer (40 mM, pH 6.8) (60/40, v/v) was used as the mobile phase. It was split at the upstream side of the injector. The flow rate in the column was $1.0 \mu \mathrm{l} / \mathrm{min}$. The
Table 1 Retention factors of first eluted enantiomers $\left(k_{1}\right)$ and separation factors $(\alpha)$ of test enantiomers on $\gamma$-CDSP and Az $\gamma$ CDSP at $25^{\circ} \mathrm{C}$

\begin{tabular}{llllll}
\hline \multirow{2}{*}{ Enantiomer } & \multicolumn{3}{c}{ Stationary phase } \\
\cline { 2 - 3 } & \multicolumn{2}{c}{$\gamma$-CDSP } & & \multicolumn{2}{c}{ Az $\gamma$-CDSP } \\
\cline { 2 - 3 } \cline { 5 - 6 } & $k_{1}$ & $\alpha$ & & $k_{1}$ & $\alpha$ \\
\hline DNSPhe & $0.250^{\mathrm{a}}\left(\mathrm{L}^{\mathrm{b}}\right)$ & $1.19^{\mathrm{a}}$ & & $0.688(\mathrm{~L})$ & 1.38 \\
DNBPEA & $0.064(R)$ & 1.13 & & $1.852(R)$ & 1.14 \\
DNBNEA & $0.228(R)$ & 1.00 & & $7.108(R)$ & 1.05 \\
Benzoin & $0.105(R)$ & 1.10 & & $1.394(S)$ & 1.07 \\
\hline
\end{tabular}

a. It was measured at $20^{\circ} \mathrm{C}$.

b. Configuration of first enantiomer.

c. Mixture of methanol and phosphate buffer $(20 \mathrm{mM}, \mathrm{pH}=6.8)$ $(40 / 60, v / v)$ was used as the mobile phase. Other LC conditions are described in the Experimental section.

prepared stationary phase was packed into UV-permeable silica capillary tubing (Supelco CElect UVT100, $100 \mathrm{~mm}$ long, 100 $\mu \mathrm{m}$ i.d.) with a flit using a slurry packing method. A Toshiba (Tokyo, Japan) Model FL4BLB black-light tube was placed in the column oven as a UV light source. For a photo-response study, the light was first irradiated on the column for a certain period, and then after the light was turned off, the HPLC measurement was started. The detection wavelength was 254 $\mathrm{nm}$. The hold-up time was measured using potassium nitrate.

\section{Results and Discussion}

Retention behaviors of the test enantiomers on Az $\gamma$-CDSP

Table 1 shows the retention factors of the first eluted enantiomers $\left(k_{1}\right)$ and their separation factors $(\alpha)$ on $\gamma$-CDSP and Az $\gamma$-CDSP. The retentions of the test enantiomers, especially, DNBPEA and DNBNEA on Az $\gamma$-CDSP, tended to be larger than those on $\gamma$-CDSP. A reversed retention order of benzoin enantiomers was found using Az $\gamma$-CDSP.

Chromatograms of Dns-D,L-Phe and $R$ - and $S$-DNBPEA are shown in Fig. 3. For $R$ - and $S$-DNBPEA in Figs. 3(c) and (d), 0.048 and 0.044 of the standard deviation $(2.6 \%$ and $2.1 \%$ of the relative standard deviation) for the retention factors from quadruplicate measurements resulted, respectively. It was also confirmed that the $k$ value of the $R$-form was smaller than that of the $S$-form for each measurement. Chiral separation of DnsPhe on $\gamma$-CDSP was not found using the racemate as the sample at $25^{\circ} \mathrm{C}(k=0.209)$. However, weaker chiral recognition than that on $\mathrm{Az} \gamma-\mathrm{CDSP}$ was found at $20^{\circ} \mathrm{C}$, which is shown in Fig.3(a). From these results, the azobenzene moiety of the Az $\gamma$-CDSP was suggested to be more effective for the retention of DNBPEA and DNBNEA and for the enantioseparation of benzoin and DnsPhe.

The chiral separation of Dns-amino acid enantiomers using native or modified $\gamma$-CD stationary phases have been reported. ${ }^{9}, 10$ For the retention mechanism of DnsPhe using $\gamma$ CDSP, the inclusion of a (dimethylamino)naphthyl moiety of the dansyl group into the cavity of $\mathrm{CD}$ is considered to be a main factor for governing the retention or enantioselectivity. ${ }^{10}$ When only the dansyl group is included in the CD cavity, the remaining benzyl group of the analyte would be located either in the proximity of the rim (L-enantiomer) or far from the rim (D-enantiomer). Under this circumstance, the inclusion complex of the D-enantiomer would be more stable because of no steric hindrance with the benzyl group; thus, the elution order of a pair of this enantiomers resulted in L- < D-enantiomer. 

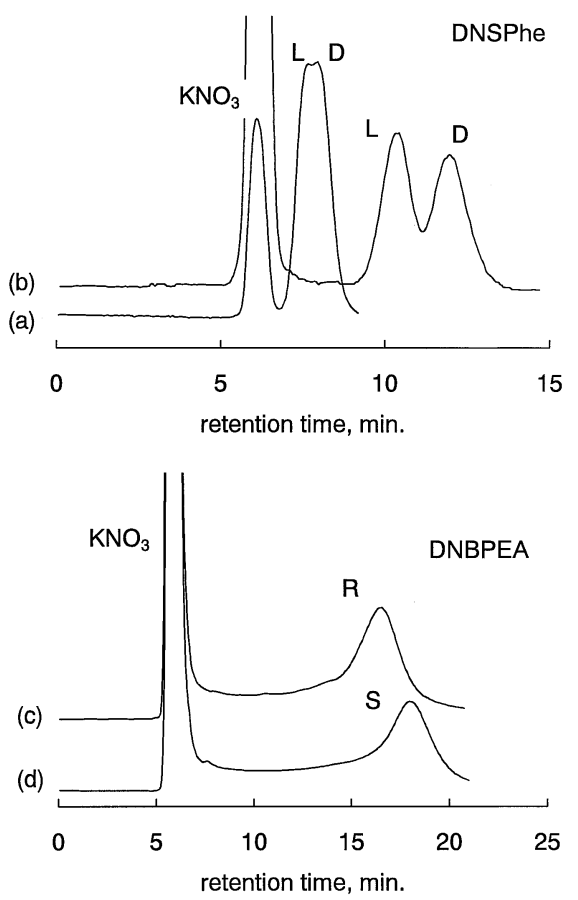

Fig. 3 Chromatograms of Dns-D,L-Phe ((a) and (b)) and R- and SDNBPEA ((c) and (d)) using $\gamma$-CDSP (a) at $20^{\circ} \mathrm{C}$ and $\mathrm{Az} \gamma-\operatorname{CDSP}((\mathrm{b})$, (c) and (d)) at $25^{\circ} \mathrm{C}$. HPLC conditions are described in the Experimental section.

On the contrary, when the dansyl group was replaced by the benzyl group, the L-enantiomer might form a more stable inclusion complex, and reversed chiral recognition would be attained. ${ }^{10}$ From the results for DnsPhe on $\gamma$-CDSP and Az $\gamma-$ CDSP in this study, it is considered that the dansyl group is preferentially included in the CD cavity of the $\gamma$-CDSP, and the azobenzene moiety of Az $\gamma$-CDSP would increase the preference because the L-enantiomer was first eluted on both stationary phases, and the enantioselectivity of Az $\gamma$-CDSP was greater than that of $\gamma$-CDSP.

It has also been reported that the enantiomers of 1phenylethylamine (NEA) could not be resolved on a $\beta$-CD stationary phase, but some separation was observed for 1-(1naphthyl)ethylamine (NEA) using a mixture of methanol and water as the mobile phase. ${ }^{8}$ This demonstrated the strict size requirements for tight binding of the solute to the $\mathrm{CD}$ cavity. The $S$-enantiomer of NEA was first eluted under this condition. In our study, $R$-DNBPEA and $R$-DNBNEA enantiomers eluted first, and the chiral separation of DNBPEA was relatively greater than that of DNBNEA on the native $\gamma$-CDSP, as shown in Table 1. Thus, it was considered that the chirality was recognized by inclusion of the DNB group of the solutes to the $\gamma$-CD cavity of the stationary phase.

The retentions of $R$-DNBPEA and $R$-DNBNEA on Az $\gamma$-CDSP were both significantly larger than those on $\gamma$-CDSP. The elution order of the enantiomers on Az $\gamma$-CDSP was the same as that on $\gamma$-CDSP. These results suggest that the $\pi$-acid (electron poor) aromatic ring of the DNB moiety of the solutes interacted with the $\pi$-bacic (electron rich) one of the azobenzene moiety in, or close to, the rim of the CD moiety of the stationary phase. The slightly greater enantioseparation of DNBNEA on Az $\gamma$ CDSP than that on $\gamma$-CDSP would be caused by the more preferential inclusion of the $\pi$-acid DNB group than the naphthyl group of the solute in the CD cavity.
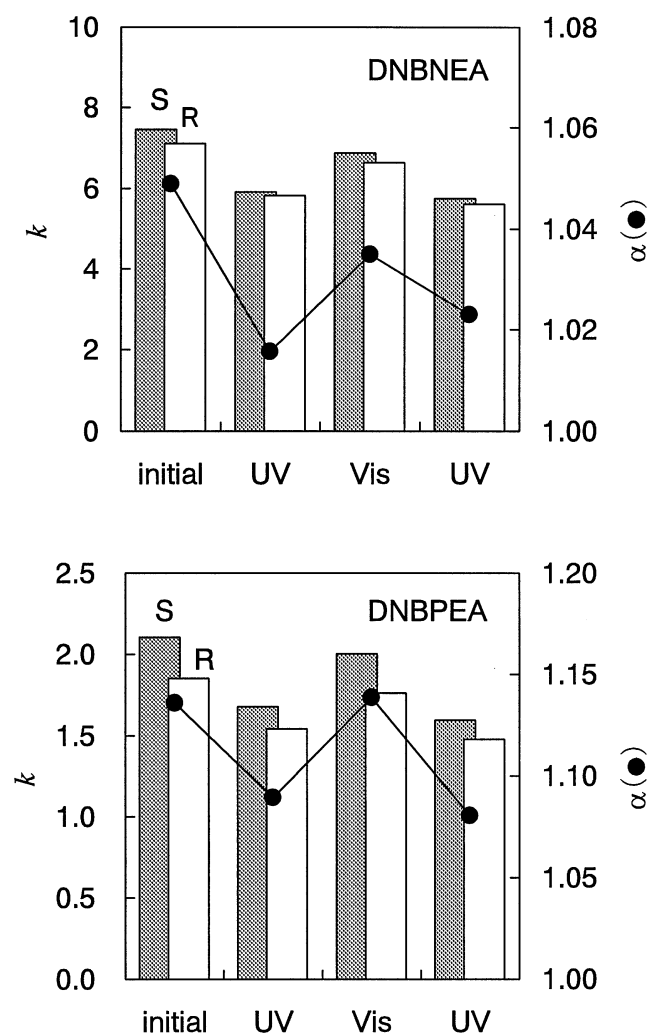

Fig. 4 Changes in the retentions (closed and open bars) and the separation factors (closed circles) of DNBPEA and DNBNEA with repeated UV and visible light irradiations using Az $\gamma$-CDSP. HPLC conditions are described in the Experimental section.

Because the benzoin molecule is the smallest of all test enantiomers, and is too small to be included alone in the $\gamma$-CD cavity, it is possible to have two or more molecules in the cavity. Furthermore, it has two aromatic rings at both ends. Consequently, a slight change in the environment, for instance, a change in the conformation of the $\mathrm{CD}$ with the immobilization of the azobenzene moiety, or the $\pi-\pi$ interaction due to the azobenzene moiety of the stationary phase, and so on, would cause changes in the enantioselectivity.

\section{Photo-response of the retention and enantioseparation on Az $\gamma$ CDSP}

The changes in the retention factors $(k)$ and separation factors $(\alpha)$ of analytes with repeated UV and visible light irradiations on Az $\gamma$-CDSP are shown in Figs. 4 and 5.

Decreases in the relatively larger $k$ and $\alpha$ values of DNBNEA and DNBPEA (Fig. 4), and an increase in the smaller $k$ but the greater $\alpha$ value of DnsPhe (Fig. 5), resulted form UV light irradiation. The retention and the chiral separation of benzoin were not changed by irradiation (Fig. 5). The initial retentions of all test samples were obtained after visible-light irradiation.

In a previous study, the retention of perylene slightly increased on the Az $\gamma$-CDSP by UV light irradiation, whereas that of pentacene significantly decreased. ${ }^{5}$ It was presumed that the trans-azobenzene moiety acts as a preventive cap of the $\mathrm{CD}$ cavity for wide and flat formed perylene, and as a spacer for the longer-shaped pentacene in filling the $\mathrm{CD}$ cavity, namely, a "capping effect" and a "spacing effect", respectively. ${ }^{5}$

If this presumption could be applied to this study, the photoresponse of DnsPhe and that of DNBPEA or DNBNEA would be caused by the "capping effect" and the "spacing effect", 

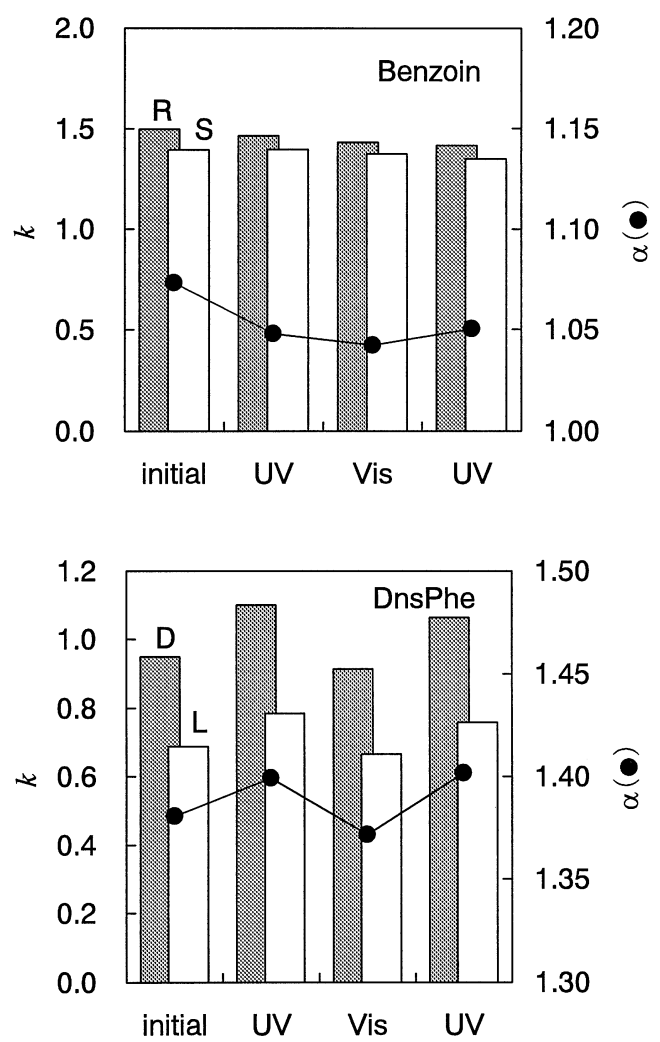

Fig. 5 Changes in the retentions (closed and open bars) and the separation factors (closed circles) of Benzoin and DnsPhe with repeated UV and visible light irradiations using Az $\gamma$-CDSP. HPLC conditions are described in the Experimental section.

respectively. However, the photo-response could not be clearly explained based only on this reason, particularly the capping effect for DnsPhe, because it seems that the molecular shapes of those solutes are not very different from those between perylene and pentacene; besides, the retention of DnsPhe on Az $\gamma$-CDSP was significantly greater than that on $\gamma$-CDSP, as shown in Table 1.

Another reason to be considered for the photo-response is a change in the $\pi-\pi$ interaction of the terminal aromatic group of the azobenzene moiety of the stationary phase.

In the case of cis-azobenzene, the $\pi$-electron density is localized on the central $\mathrm{N}=\mathrm{N}$ bond as compared to greater $\pi$ conjugation in trans-azobenze, leading to an increased doublebond character for $\mathrm{N}=\mathrm{N} .{ }^{14}$ The $\pi$ system of the adjacent aromatic ring is electronegative with respect to that of cisazobenzene based on a molecular-orbital analysis. ${ }^{15}$ The $\pi$ electron in the aromatic ring of the azobenzene moiety might become relatively poor due to trans to cis photo-isomerization with UV light irradiation.

If the $\pi$-electron density in the terminal aromatic group of the azobenzene moiety decreases upon UV light irradiation, an increase in the $\pi$ - $\pi$ interaction with the somewhat $\pi$-basic (electron rich) Dns group of DnsPhe and a decrease of that with the $\pi$-acidic (electron poor) DNB group of DNBPEA or DNBNEA after UV light irradiation could be predicted. This prediction is consistent with the photo-responsive obtained results.

The retention characteristics of benzoin would result by compensation of the increase in the host-guest interaction and the decrease in the $\pi$ - $\pi$ interaction.

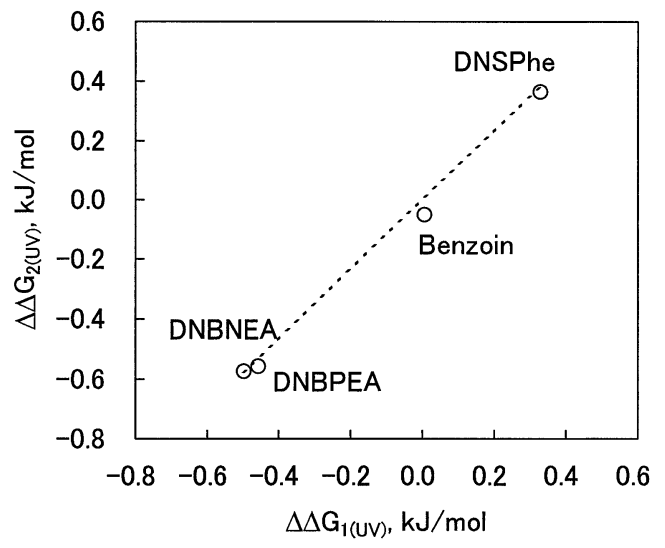

Fig. 6 Relationship between the change in the Gibbs free energy for the retention of first eluted enantiomers $\left(\Delta \Delta G_{1(\mathrm{UV})}\right)$ and those of latter eluted ones $\left(\Delta \Delta G_{2(\mathrm{UV})}\right)$ by UV light irradiation using $\mathrm{Az} \gamma-$ CDSP.

Thermodynamic study of the photo-response of the retention and enantioselectivity on Az $\gamma-C D S P$

The photo-response was evaluated using the difference in the Gibbs free energy for the retentions of the enantiomers due to UV light irradiation $\left(\Delta \Delta G_{\mathrm{r}(\mathrm{UV})}\right)$. It was calculated by using

$$
\Delta \Delta G_{\mathrm{r}(\mathrm{UV})}=R T \ln \left(k_{(\mathrm{UV})} / k\right),
$$

where $k$ and $k_{(\mathrm{UV})}$ represent the retention factors before and after UV light irradiation. For each test enantiomer,

$$
\begin{aligned}
& \Delta \Delta G_{\mathrm{r} 1(\mathrm{UV})}=R T \ln \left(k_{1(\mathrm{UV})} / k_{1}\right), \\
& \Delta \Delta G_{\mathrm{r} 2(\mathrm{UV})}=R T \ln \left(k_{2(\mathrm{UV})} / k_{2}\right),
\end{aligned}
$$

where $k_{1}$ represents the first elute enantiomer. The correlation of $\Delta \Delta G_{\mathrm{r} 1(\mathrm{UV})}$ and $\Delta \Delta G_{\mathrm{r} 2(\mathrm{UV})}$ is shown in Fig. 6. A good linear relationship was found,

$$
\Delta \Delta G_{\mathrm{r} 2(\mathrm{UV})}=1.17 \Delta \Delta G_{\mathrm{r} 1(\mathrm{UV})} \quad(r=0.996) .
$$

The difference in the Gibbs free energy between the enantiomers could be calculated by

$$
\Delta \Delta G_{\mathrm{c}}=R T \ln \alpha=R T \ln \left(k_{2} / k_{1}\right) \quad\left(k_{2}>k_{1}\right) .
$$

For Az $\gamma$-CDSP after UV light irradiation,

$$
\Delta \Delta G_{\mathrm{c}(\mathrm{UV})}=R T \ln \left(k_{2(\mathrm{UV})} / k_{1(\mathrm{UV})}\right) .
$$

When the photo-response of the chiral separation was defined by Eq. (5) based on the result of Eq. (4),

$$
\begin{aligned}
\Delta \Delta \Delta G_{\mathrm{c}(\mathrm{UV})} & =\Delta \Delta G_{\mathrm{c}(\mathrm{UV})}-\Delta \Delta G_{\mathrm{c}}=\Delta \Delta G_{\mathrm{r} 2(\mathrm{UV})}-\Delta \Delta G_{\mathrm{r} 1(\mathrm{UV})} \\
& =0.17 \Delta \Delta G_{\mathrm{r} 1(\mathrm{UV})} .
\end{aligned}
$$

Equation (7) indicates that the change in the energy for the separation of enantiomers with UV light irradiation corresponds to $17 \%$ of the energy required for the change in their retention. The change in separation factor after UV light irradiation could be estimated from the retention change using this equation. 


\section{Conclusion}

The retentions and the chiral separations of several enantiomers on $\gamma$-CDSP and Az $\gamma$-CDSP were compared. The photoresponse of the retention behaviors on Az $\gamma$-CDSP were evaluated.

It was clarified that the azobenzene moiety of Az $\gamma$-CDSP was effective for the retention, and that the photo-response was differently affected by the molecular structure of the analyte. A better resolution of the enantiomers could be chosen through a comparison before and after UV light irradiation for a specific period on Az $\gamma$-CDSP.

\section{References}

1. K. Ishihara, N. Negishi, and I. Shinohara, J. Appl. Polym. Sci., 1982, 27, 1897 .

2. K. Ishihara, S. Kato, and I. Shinohara, J. Appl. Polym. Sci., 1982, 27, 4273

3. K. Ishihara, M. Kim, I. Shinohara, T. Okano, K. Kataoka, and Y. Sakurai, J. Appl. Polym. Sci., 1983, 28, 1321.

4. H.Ishihara, T. Nakagama, and T. Hobo, J. High Resol. Chromatogr., 1998, 21, 25.

5. T. Nakagama, K. Hirasawa, K. Uchiyama, and T. Hobo,
Anal. Sci., 2001, 17, 119.

6. A. Berthod, C. D. Chang, and D. W. Armstrong, Talanta, 1993, 40, 1367.

7. S. H. Lee, A. Berthod, and D. W. Armstrong, $J$. Chromatogr., 1992, 603, 83.

8. W. L. Hinze, T. E. Riehl, D. W. Armstrong, W. DeMond, A. Alak, and T. Ward, Anal. Chem., 1985, 57, 237.

9. T. Araki, Y. Kashiwamoto, S. Tsunoi, and M. Tanaka, J. Chromatogr. A, 1999, 845, 455.

10. K. Fujimura, S. Suzuki, K. Hayashi, and S. Masuda, Anal. Chem., 1990, 62, 2198.

11. R. A. Menges and D. W. Armstrong, in "Chiral Separations by Liquid Chromatography", ed. S. Ahuja, 1991, Vol. 471 of ACS Symposium Series, Am. Chem. Soc., Washington, D.C., 67.

12. J. Snopek, E. Smolkova-Keulemansova, T. Cserhati, K. H. Gahm, and A. Stalcup, in "Cyclodextrins", ed. J. Szejtli and T. Osa, 1996, Vol. 3 of Comprehensive Supermolecular Chemistry, Elsevier Sci. Ltd., Oxford, 555.

13. A. Ueno, Y. Tomita, and T. Osa, Tetrahedron Lett., 1983, 24,5245 .

14. N. Biswas and S. Umapathy, J. Phys. Chem. A, 1997, 101, 5555.

15. E. V. Brown and G. R. Granneman, J. Am. Chem. Soc., $\mathbf{1 9 7 5}, 97,621$ 